\title{
Palladium-Catalyzed Silylation of Aryl Chlorides with Hexamethyldisilane
}

\author{
Eric McNeill, Timothy E. Barder and Stephen L. Buchwald* \\ Department of Chemistry, Massachusetts Institute of Technology, \\ Cambridge, Massachusetts 02139 \\ sbuchwal@mit.edu
}

\section{Supporting Information}

General considerations. All reactions were carried out under an argon atmosphere. Anhydrous 1,4-dioxane and $N, N$-dimethylformamide were purchased from Aldrich and used without further purification. All starting materials are commercially available and were used without further purification. KF was purchased from Aldrich. The bulk of KF was stored in a nitrogen-filled glovebox; however, 1-2 g portions were removed from the glovebox, stored in a benchtop dessicator and weighed in the air. Anhydrous LiOAc was purchased from Aldrich and stored in a desiccator.

Analytical Methods. All new compounds were characterized by ${ }^{1} \mathrm{H},{ }^{13} \mathrm{C}$ NMR and IR spectroscopy, elemental analysis, and melting point where appropriate. Known compounds were characterized by ${ }^{1} \mathrm{H}$ and ${ }^{13} \mathrm{C}$ NMR and melting point where appropriate. Melting points were determined using a Mel-Temp II (Laboratory Devices) and are uncorrected. NMR spectra were recorded using a Varian Mercury 300 instrument. All ${ }^{1} \mathrm{H}$ NMR chemical shifts were reported in parts per million (ppm) relative to the residual protonated solvent peak (7.26 ppm for $\mathrm{CDCl}_{3}$ ). Proton-decoupled ${ }^{13} \mathrm{C}$ NMR chemical shifts were reported relative to the solvent peak (77.23 ppm for $\mathrm{CDCl}_{3}$ ). Infrared spectra were recorded on a Perkin-Elmer Model 2000 FT-IR using $\mathrm{NaCl}$ plates (thin film). Elemental analyses were preformed by Atlantic Microlabs Inc., Norcros, GA. Gas chromatographic analyses were performed on Hewlett-Packard 6890 gas chromatography instrument with a FID detector using $25 \mathrm{~m} \times 0.20 \mathrm{~mm}$ capillary column with cross-linked methyl siloxane as a stationary phase. The yields in Tables 2, 4 and 5 refer to 
isolated yields of compounds estimated to be $\geq 95 \%$ pure as determined by combustion analysis or ${ }^{1} \mathrm{H}$ NMR and are the average of two runs. The procedures described in this Supporting Information are representative, and hence the yields may differ slightly from those reported in Tables 2 and 4.

Cross-coupling of aryl chlorides with hexamethyldisilane, procedure A (electron-rich aryl chlorides). A screw-cap vial with Teflon septum was charged with a magnetic stir bar, tris(dibenzylideneacetone)dipalladium(0), 2-(dicyclohexylphosphino)biphenyl (1b, in a 3:1 ratio of $\mathbf{1 b}: \mathrm{Pd})$, and anhydrous potassium fluoride $(5 \mathrm{mmol}, 290 \mathrm{mg})$. The vial was sealed, evacuated and filled with argon ( 2 cycles). Aryl chloride $(1 \mathrm{mmol})$, dioxane $(3 \mathrm{~mL})$, hexamethyldisilane $(1.2-1.8 \mathrm{mmol})$, and water $(1-2 \mathrm{mmol})$ were added via syringe. Solid aryl chlorides were added prior to sealing the vial. The reaction mixture was stirred at $100{ }^{\circ} \mathrm{C}$ for $24 \mathrm{~h}$, then allowed to cool to room temperature. The reaction was diluted with $\mathrm{H}_{2} \mathrm{O}(3 \mathrm{~mL})$ and extracted with hexanes $(4 \times 3 \mathrm{~mL})$. The combined organic layers were dried over $\mathrm{MgSO}_{4}$, concentrated in vacuo, and purified by silica gel chromatography.

\section{Cross-coupling of aryl chlorides with hexamethyldisilane, procedure B (electron-deficient} aryl chlorides). A screw-cap vial with Teflon septum was charged with a magnetic stir bar, tris(dibenzylideneacetone)dipalladium(0) (0.015 mmol, $13.7 \mathrm{mg}), 2^{\prime}$-(dimethylamino)-2-(di-tertbutylphosphino)biphenyl (1d, $0.09 \mathrm{mmol}, 31.5 \mathrm{mg}$ ), and anhydrous lithium acetate (5 mmol, 330 $\mathrm{mg})$. The vial was sealed, evacuated and filled with argon (2 cycles). Aryl chloride ( $1 \mathrm{mmol})$, water $(2 \mathrm{mmol}, 36 \mu \mathrm{L})$, hexamethyldisilane $(1.2 \mathrm{mmol}, 250 \mu \mathrm{L})$, and $N, N$-dimethylformamide (3 $\mathrm{mL}$ ) were added via syringe. Solid aryl chlorides were added prior to sealing the vial. The reaction mixture was stirred at $100{ }^{\circ} \mathrm{C}$ for $24 \mathrm{~h}$, then allowed to cool to room temperature. The reaction was diluted with $\mathrm{H}_{2} \mathrm{O}(3 \mathrm{~mL})$ and extracted with hexanes $(4 \times 3 \mathrm{~mL})$. The combined organic layers were dried over $\mathrm{MgSO}_{4}$, concentrated in vacuo, and purified by silica gel chromatography.

One-pot sequential trimethylsilylation/iodination of electron-rich aryl chlorides. A screwcap vial with Teflon septum was charged with a magnetic stir bar, tris(dibenzylideneacetone)dipalladium(0) (0.015 mmol, $13.7 \mathrm{mg})$, 2-(dicyclohexylphosphino)- 
biphenyl (1b, $0.09 \mathrm{mmol}, 31.5 \mathrm{mg}$ ), and anhydrous potassium fluoride (5 mmol, $290 \mathrm{mg})$. The vial was sealed, evacuated and filled with argon (2 cycles). Aryl chloride (1.0 mmol), hexamethyldisilane (1.2 mmol, $250 \mu \mathrm{L})$, water $(2 \mathrm{mmol}, 36 \mu \mathrm{L})$, and dioxane $(3 \mathrm{~mL})$ were added via syringe. The reaction was stirred at $100{ }^{\circ} \mathrm{C}$ for $24 \mathrm{~h}$, then allowed to cool to room temperature. Iodine monochloride $\left(1.5 \mathrm{mmol}\right.$, as a $1.0 \mathrm{M}$ solution in $\left.\mathrm{CH}_{2} \mathrm{Cl}_{2}\right)$ was added via syringe, and the reaction mixture was stirred at room temperature for $4 \mathrm{~h}$. The reaction was diluted with a $1 \mathrm{M}$ solution of $\mathrm{Na}_{2} \mathrm{~S}_{2} \mathrm{O}_{3}(3 \mathrm{~mL})$ and extracted with hexanes $(4 \times 3 \mathrm{~mL})$. The combined organic layers were dried over $\mathrm{MgSO}_{4}$, concentrated in vacuo, and purified by column chromatography.

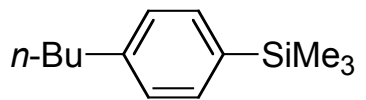

(4-n-Butylphenyl)trimethylsilane (Table 2, entry 1). ${ }^{[1]}$ Following procedure A, a mixture of 4chloro- $n$-butylbenzene (1.0 mmol, $158 \mathrm{mg}), \mathrm{KF}(5 \mathrm{mmol}, 290 \mathrm{mg})$, hexamethyldisilane $(1.2$ mmol, $250 \mu \mathrm{L}), \mathrm{Pd}_{2} \mathrm{dba}_{3}(0.0075 \mathrm{mmol}, 7 \mathrm{mg}), \mathbf{1 b}(0.045 \mathrm{mmol}, 16.5 \mathrm{mg})$ and $\mathrm{H}_{2} \mathrm{O}$ (2 mmol, 36 $\mu \mathrm{L})$ in dioxane $(3 \mathrm{~mL})$ was heated to $100{ }^{\circ} \mathrm{C}$ with stirring for $24 \mathrm{~h}$. The crude product was purified via column chromatography on silica gel (hexanes) to afford the title compound as a colorless oil (185 mg, 90\%). ${ }^{1} \mathrm{H}$ NMR $\left(300 \mathrm{MHz}, \mathrm{CDCl}_{3}\right): \delta 7.45(\mathrm{~d}, J=8 \mathrm{~Hz}, 2 \mathrm{H}), 7.19(\mathrm{~d}, J=$ $8 \mathrm{~Hz}, 2 \mathrm{H}), 2.60$ (t, J= $7 \mathrm{~Hz}, 2 \mathrm{H}), 1.61(\mathrm{~m}, 2 \mathrm{H}), 1.37(\mathrm{dt}, J=7 \mathrm{~Hz}, 8 \mathrm{~Hz}, 2 \mathrm{H}), 0.94(\mathrm{t}, J=7 \mathrm{~Hz})$, $0.27(\mathrm{~s}, 9 \mathrm{H}) .{ }^{13} \mathrm{C} \mathrm{NMR}\left(75 \mathrm{MHz}, \mathrm{CDCl}_{3}\right): \delta 143.8,137.3,133.6,128.2,35.9,33.8,22.7,14.2$, 0.8. A copy of the ${ }^{1} \mathrm{H}$ NMR spectrum is provided below. 

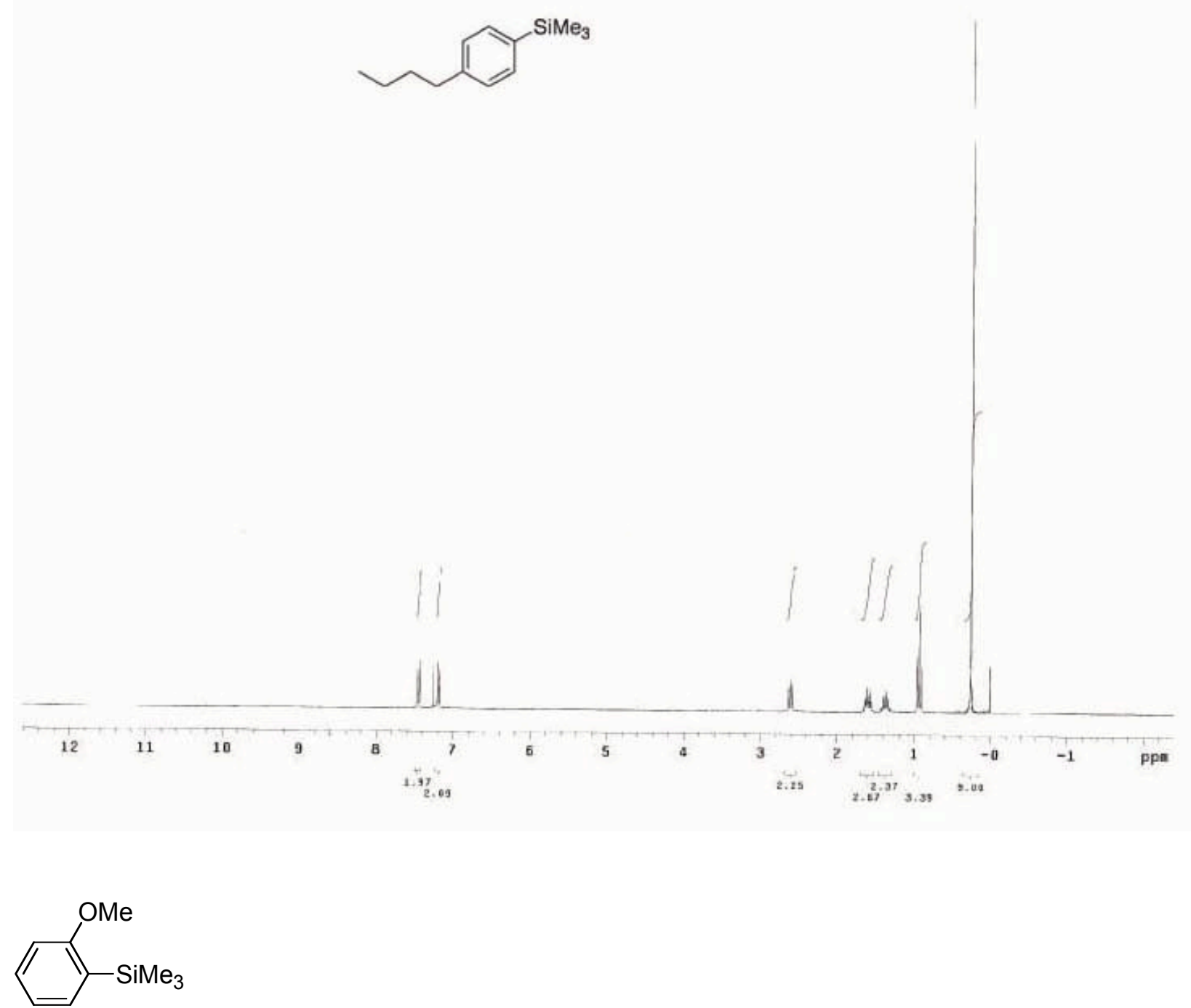

2-Trimethylsilylanisole (Table 2, entry 2). ${ }^{[2]}$ Following procedure A, a mixture of 2chloroanisole (1.0 mmol, $143 \mathrm{mg}$ ), KF (5 mmol, $290 \mathrm{mg}$ ), hexamethyldisilane (1.5 mmol, 313 $\mu \mathrm{L}), \mathrm{Pd}_{2} \mathrm{dba}_{3}(0.015 \mathrm{mmol}, 13.7 \mathrm{mg}), \mathbf{1 b}(0.09 \mathrm{mmol}, 31.5 \mathrm{mg})$ in dioxane $(3 \mathrm{~mL})$ was heated to $100{ }^{\circ} \mathrm{C}$ with stirring for $24 \mathrm{~h}\left(\mathrm{H}_{2} \mathrm{O}\right.$ omitted for this reaction). The crude product was purified via column chromatography on silica gel (hexanes) to afford the title compound as a colorless oil (143 mg, 79\%). ${ }^{1} \mathrm{H}$ NMR (300 MHz, $\left.\mathrm{CDCl}_{3}\right): \delta$ 7.42-7.35 (m, 2H), $6.97(\mathrm{t}, J=7 \mathrm{~Hz}, 1 \mathrm{H}), 6.85$ $(\mathrm{d}, J=8 \mathrm{~Hz}, 1 \mathrm{H}), 3.82(\mathrm{~s}, 3 \mathrm{H}), 0.29(\mathrm{~s}, 9 \mathrm{H}) .{ }^{13} \mathrm{C} \mathrm{NMR}\left(75 \mathrm{MHz}, \mathrm{CDCl}_{3}\right): \delta$ 164.6, 135.2, 131.0, 128.1, 120.6, 109.7, 55.2, -0.7. A copy of the ${ }^{1} \mathrm{H}$ NMR spectrum is provided below. 

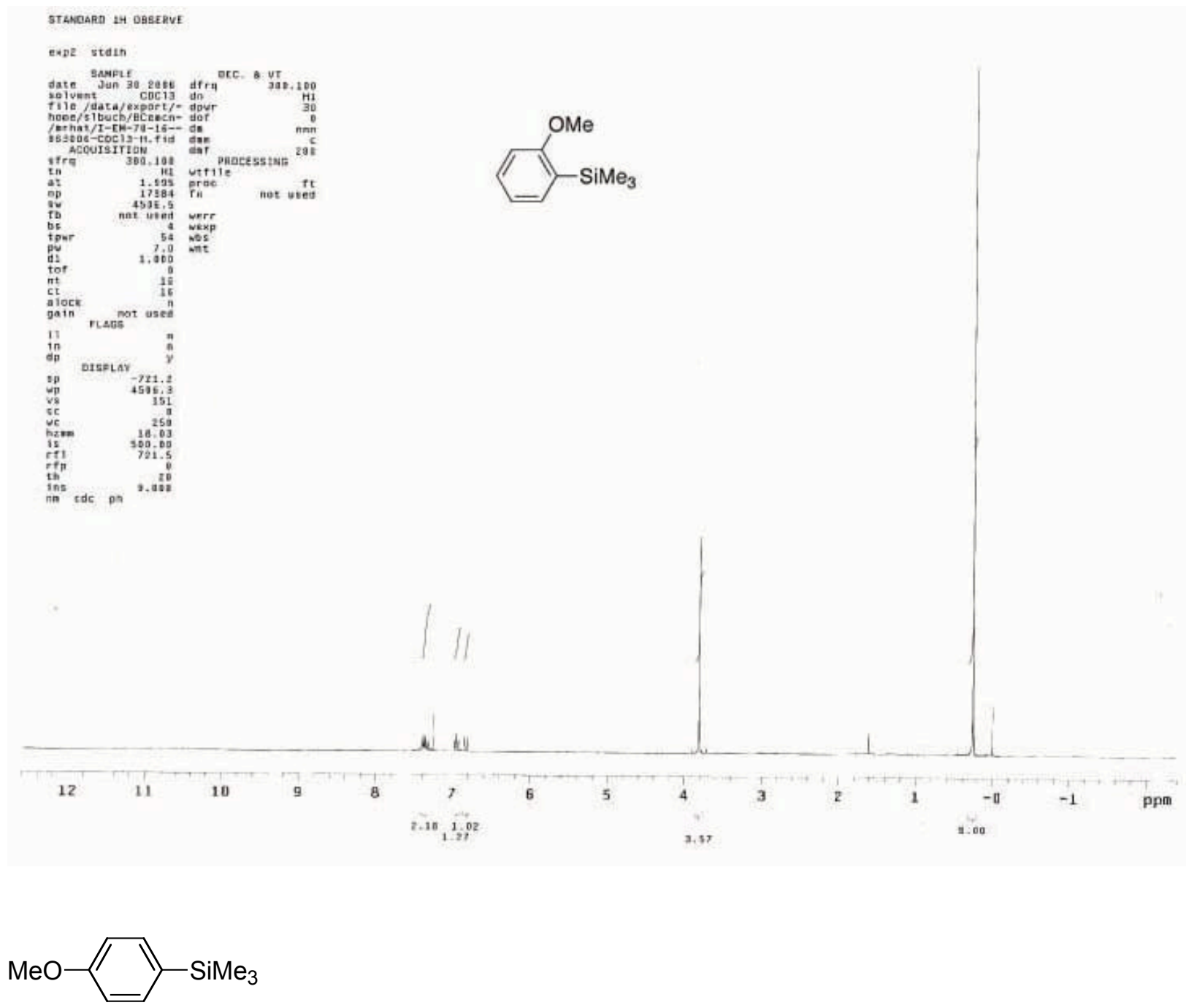

4-Trimethylsilylanisole (Table 2, entry 3). ${ }^{[2]}$ Following procedure A, a mixture of 4chloroanisole (1.0 mmol, $143 \mathrm{mg})$, KF (5 mmol, $290 \mathrm{mg})$, hexamethyldisilane (1.2 mmol, 250 $\mu \mathrm{L}), \mathrm{Pd}_{2} \mathrm{dba}_{3}(0.01 \mathrm{mmol}, 9.4 \mathrm{mg}), \mathbf{1 b}(0.06 \mathrm{mmol}, 22 \mathrm{mg})$ and $\mathrm{H}_{2} \mathrm{O}(2 \mathrm{mmol}, 36 \mu \mathrm{L})$ in dioxane (3 mL) was heated to $100{ }^{\circ} \mathrm{C}$ with stirring for $24 \mathrm{~h}$. The crude product was purified via column chromatography on silica gel (hexanes) to afford the title compound as a colorless oil (158 $\mathrm{mg}$, 96\%). ${ }^{1} \mathrm{H}$ NMR (300 MHz, $\left.\mathrm{CDCl}_{3}\right): \delta 7.45$ (d, $\left.J=8 \mathrm{~Hz}, 2 \mathrm{H}\right), 6.91$ (d, $\left.J=8 \mathrm{~Hz}, 2 \mathrm{H}\right), 3.81$ (s, $3 \mathrm{H}), 0.24(\mathrm{~s}, 9 \mathrm{H}) .{ }^{13} \mathrm{C} \mathrm{NMR}\left(75 \mathrm{MHz}, \mathrm{CDCl}_{3}\right) \delta 160.2,134.7,131.3,113.5,55.0,-1.0$. A copy of the ${ }^{1} \mathrm{H}$ NMR spectrum is provided below. 


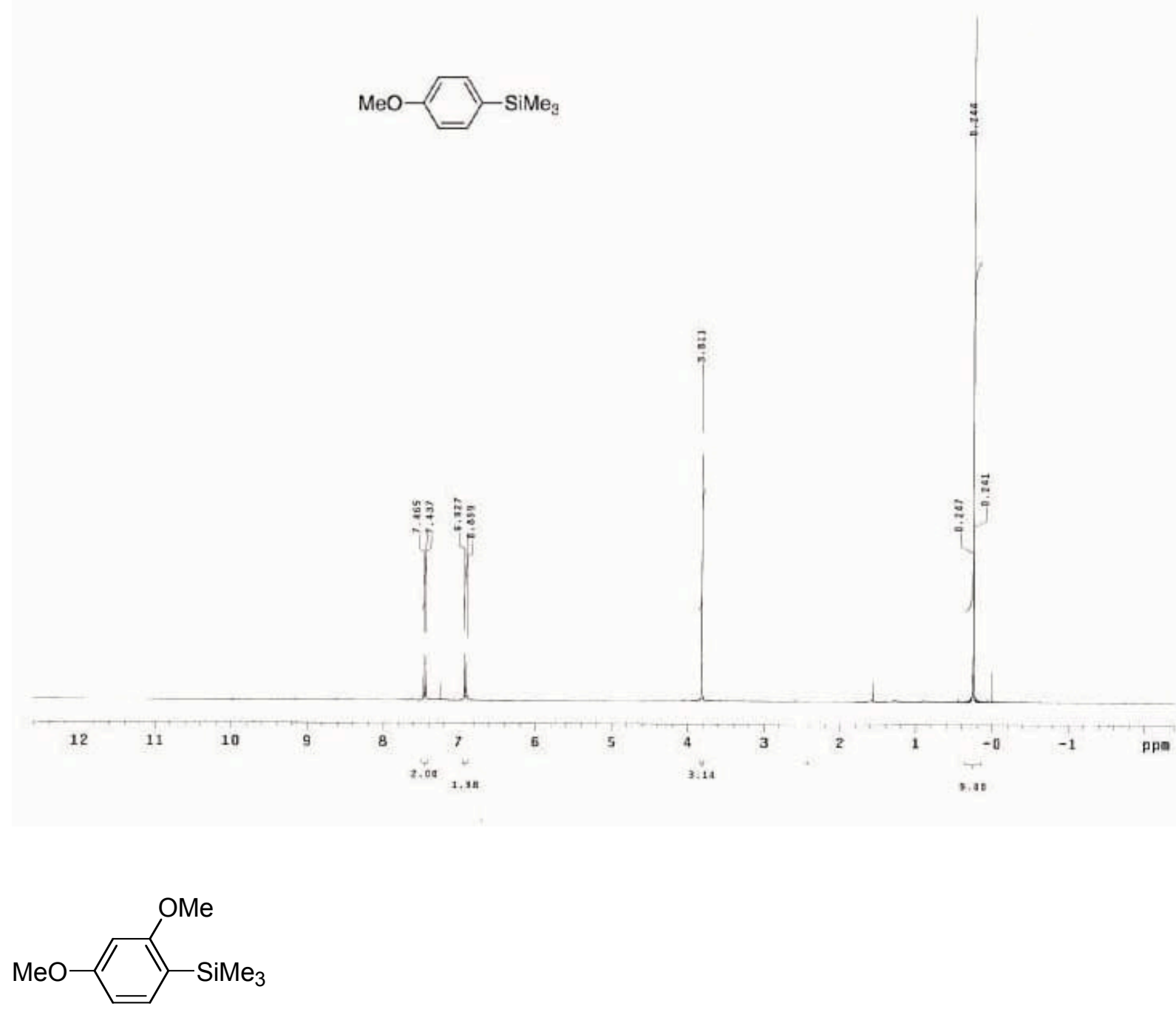

(2,4-Dimethoxyphenyl)trimethylsilane (Table 2, entry 4). Following procedure A, a mixture of 1-chloro-2,4-dimethoxybenzene (1.0 mmol, $173 \mathrm{mg}), \quad \mathrm{KF} \quad(5 \mathrm{mmol}, 290 \mathrm{mg})$, hexamethyldisilane (1.8 mmol, $375 \mu \mathrm{L}), \mathrm{Pd}_{2} \mathrm{dba}_{3}(0.015 \mathrm{mmol}, 13.7 \mathrm{mg}), \mathbf{1 b}$ (0.09 mmol, 31.5 $\mathrm{mg})$ in dioxane $(3 \mathrm{~mL})$ was heated to $100{ }^{\circ} \mathrm{C}$ with stirring for $24 \mathrm{~h}\left(\mathrm{H}_{2} \mathrm{O}\right.$ omitted for this reaction). The crude product was purified via column chromatography on silica gel (1:20 ethyl acetate:hexanes) to afford the title compound as a colorless oil (196 mg, 90\%). ${ }^{1} \mathrm{H}$ NMR (300 $\left.\mathrm{MHz}, \mathrm{CDCl}_{3}\right): \delta 6.95(\mathrm{~d}, J=3 \mathrm{~Hz}, 1 \mathrm{H}), 6.86(\mathrm{dd}, J=9 \mathrm{~Hz}, 3 \mathrm{~Hz}, 2 \mathrm{H}), 6.77$ (d, $\left.J=9 \mathrm{~Hz}, 1 \mathrm{H}\right)$, $3.78(\mathrm{~s}, 3 \mathrm{H}), 3.77(\mathrm{~s}, 3 \mathrm{H}), 0.27(\mathrm{~s}, 9 \mathrm{H}) .{ }^{13} \mathrm{C} \mathrm{NMR}\left(75 \mathrm{MHz}, \mathrm{CDCl}_{3}\right): \delta 153.6,129.7,121.4$, 114.9, 114.6, 110.6, 56.0, 55.9, -0.8. IR (neat): 2996, 2952, 2901, 2832, 1583, 1509, 1480, 1400, 1274, 1220, 1178, 1048, 1026, 885, 839, $726 \mathrm{~cm}^{-1}$. Anal. Calcd. for $\mathrm{C}_{11} \mathrm{H}_{18} \mathrm{O}_{2} \mathrm{Si}: \mathrm{C} 62.81 \%, \mathrm{H}$ 8.63\%. Found: C $63.02 \%, \mathrm{H} 8.69 \%$. 


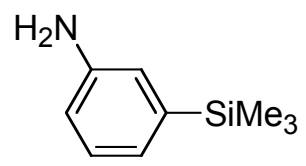

3-Trimethylsilylaniline (Table 2, entry 5). Following procedure A, a mixture of 3chloroaniline (1.0 mmol, $128 \mathrm{mg})$, KF (5 mmol, $290 \mathrm{mg})$, hexamethyldisilane $(1.8 \mathrm{mmol}, 375$ $\mu \mathrm{L}), \mathrm{Pd}_{2} \mathrm{dba}_{3}(0.01 \mathrm{mmol}, 9.4 \mathrm{mg}), \mathbf{1 b}(0.06 \mathrm{mmol}, 22 \mathrm{mg})$, and $\mathrm{H}_{2} \mathrm{O}(1 \mathrm{mmol}, 18 \mu \mathrm{L})$ in dioxane (3 mL) was heated to $100{ }^{\circ} \mathrm{C}$ with stirring for $24 \mathrm{~h}$. The crude product was purified via column chromatography on silica gel (1:20 ethyl acetate:hexanes) to afford the title compound as a pale yellow oil (132 mg, 80\%). ${ }^{1} \mathrm{H}$ NMR $\left(300 \mathrm{MHz}, \mathrm{CDCl}_{3}\right): \delta 7.17(\mathrm{t}, J=7 \mathrm{~Hz}, 1 \mathrm{H}), 6.93(\mathrm{dt}, J=7$ Hz, $1 \mathrm{~Hz}, 1 \mathrm{H}), 6.85$ (d, $J=1 \mathrm{~Hz}, 1 \mathrm{H}), 6.69$ (ddd, $J=8 \mathrm{~Hz}, 3 \mathrm{~Hz}, 1 \mathrm{~Hz}, 1 \mathrm{H}), 3.64$ (bs, 2H), 0.25 (s, 9H). ${ }^{13} \mathrm{C} \mathrm{NMR}\left(75 \mathrm{MHz}, \mathrm{CDCl}_{3}\right): \delta 145.8,141.8,128.9,123.7,120.1,115.9,-0.9$. IR (neat): 3452, 3372, 2955, 1618, 1591, 1485, 1426, 1248, 1128, 1093, 898, 837, 753, 690, $621 \mathrm{~cm}^{-1}$ Anal. Calcd. for $\mathrm{C}_{9} \mathrm{H}_{15} \mathrm{NSi}$ : C $65.39 \%$, H 9.15\%. Found: C 65.32\%, H 9.12\%.

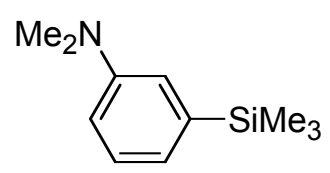

3-Trimethylsilyl- $N, N$-dimethylaniline (Table 2, entry 6). Following procedure A, a mixture of 3-chloro- $N, N$-dimethylaniline (1.0 mmol, $156 \mathrm{mg}), \mathrm{KF}$ (5 mmol, $290 \mathrm{mg})$, hexamethyldisilane (1.2 mmol, $250 \mu \mathrm{L}), \mathrm{Pd}_{2} \mathrm{dba}_{3}(0.01 \mathrm{mmol}, 9.4 \mathrm{mg}), \mathbf{1 b}(0.06 \mathrm{mmol}, 22 \mathrm{mg})$, and $\mathrm{H}_{2} \mathrm{O}(2 \mathrm{mmol}$, $36 \mu \mathrm{L})$ in dioxane $(3 \mathrm{~mL})$ was heated $100{ }^{\circ} \mathrm{C}$ with stirring for $24 \mathrm{~h}$. The crude product was purified via column chromatography on silica gel (1:20 ethyl acetate:hexanes) to afford the title compound as a colorless oil (181 mg, 94\%). ${ }^{1} \mathrm{H} \mathrm{NMR}\left(300 \mathrm{MHz}, \mathrm{CDCl}_{3}\right): \delta 7.26(\mathrm{dd}, \mathrm{J}=8 \mathrm{~Hz}$, $7 \mathrm{~Hz}, 1 \mathrm{H}), 6.90(\mathrm{~m}, 2 \mathrm{H}), 6.76(\mathrm{ddd}, J=8 \mathrm{~Hz}, 3 \mathrm{~Hz}, 2 \mathrm{~Hz}, 1 \mathrm{H}), 3.00(\mathrm{~s}, 6 \mathrm{H}), 0.27(\mathrm{~s}, 9 \mathrm{H}) .{ }^{13} \mathrm{C}$ NMR (75 MHz, $\left.\mathrm{CDCl}_{3}\right): \delta 150.1,141.2,128.7,121.9,117.5,113.6,40.9,-0.8$. IR (neat): 2955, $2985,2800,1588,1566,1491,1417,1346,1247,1183,1122,993,857,840,753,690 \mathrm{~cm}^{-1}$. A satisfactory elemental analysis could not be obtained for this compound. Copies of the ${ }^{1} \mathrm{H}$ and ${ }^{13} \mathrm{C}$ NMR spectra are provided below. 

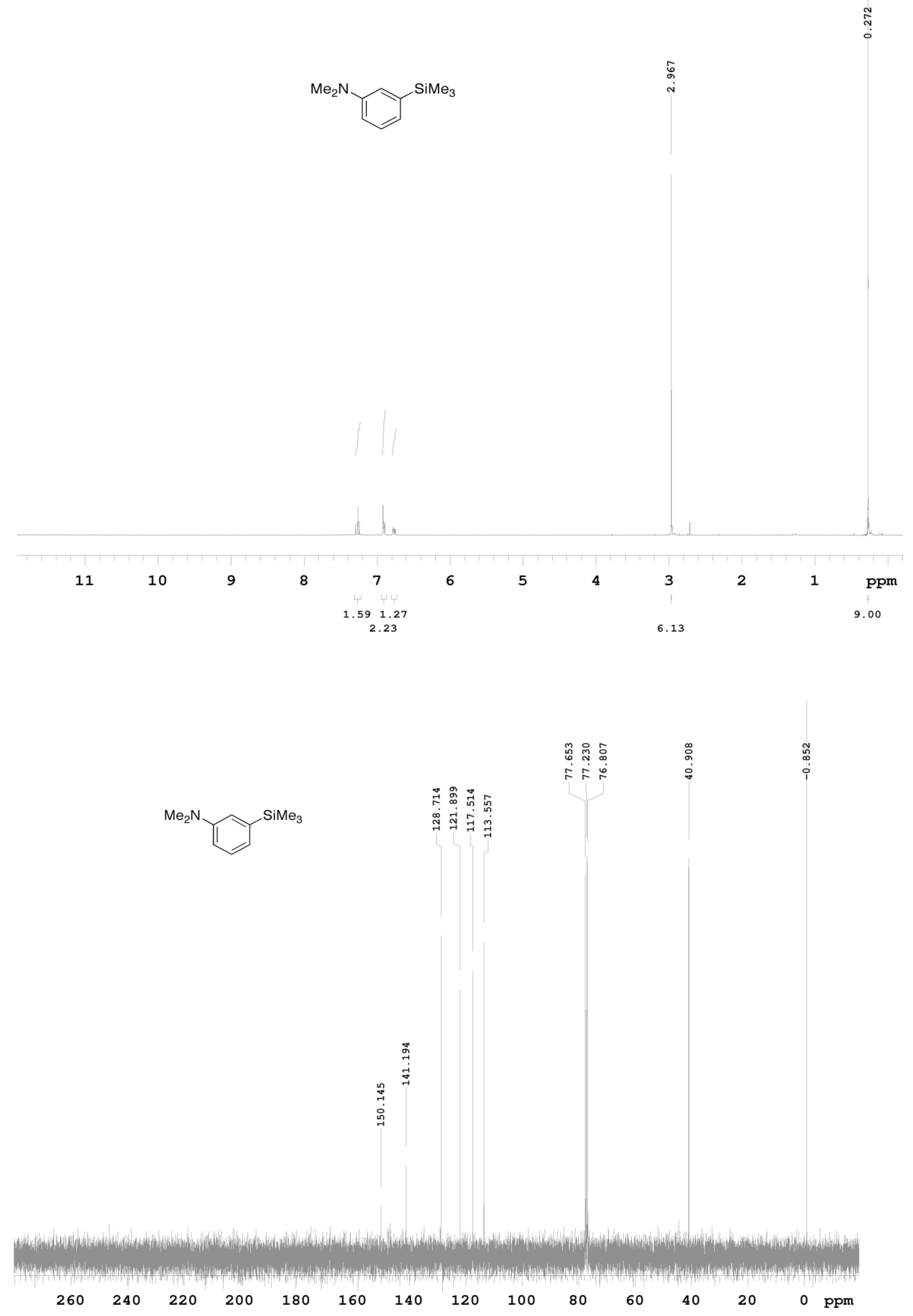


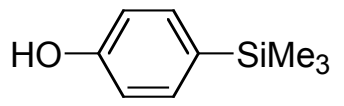

4-Trimethylsilylphenol (Table 2, entry 7). ${ }^{[2]}$ Following procedure A, a mixture of 4chlorophenol (1.0 mmol, $129 \mathrm{mg})$, KF (5 mmol, $290 \mathrm{mg})$, hexamethyldisilane (1.2 mmol, 250 $\mu \mathrm{L}), \mathrm{Pd}_{2} \mathrm{dba}_{3}(0.015 \mathrm{mmol}, 13.7 \mathrm{mg}), \mathbf{1 b}(0.09 \mathrm{mmol}, 31.5 \mathrm{mg})$, and $\mathrm{H}_{2} \mathrm{O}(2 \mathrm{mmol}, 36 \mu \mathrm{L})$ in dioxane $(3 \mathrm{~mL})$ was heated $100{ }^{\circ} \mathrm{C}$ with stirring for $24 \mathrm{~h}$. The crude product was purified via column chromatography on silica gel (1:25 ethyl acetate:hexanes) to afford the title compound as a colorless solid $(120 \mathrm{mg}, 72 \%)$. MP: $73-75{ }^{\circ} \mathrm{C}$ (lit. 74-75 $\left.{ }^{\circ} \mathrm{C}\right) .{ }^{1} \mathrm{H} \mathrm{NMR}\left(300 \mathrm{MHz}, \mathrm{CDCl}_{3}\right): \delta$ $7.42(\mathrm{dt}, J=8 \mathrm{~Hz}, 2 \mathrm{~Hz}, 2 \mathrm{H}), 6.86(\mathrm{dt}, J=7 \mathrm{~Hz}, 1 \mathrm{~Hz}, 2 \mathrm{H}), 5.04$ (bs, 1H), $0.26(\mathrm{~s}, 9 \mathrm{H}) .{ }^{13} \mathrm{C}$ NMR $\left(75 \mathrm{MHz}, \mathrm{CDCl}_{3}\right): \delta 156.3,135.2,131.9,115.1,-0.7$. A copy of the ${ }^{1} \mathrm{H}$ NMR spectrum is provided below.

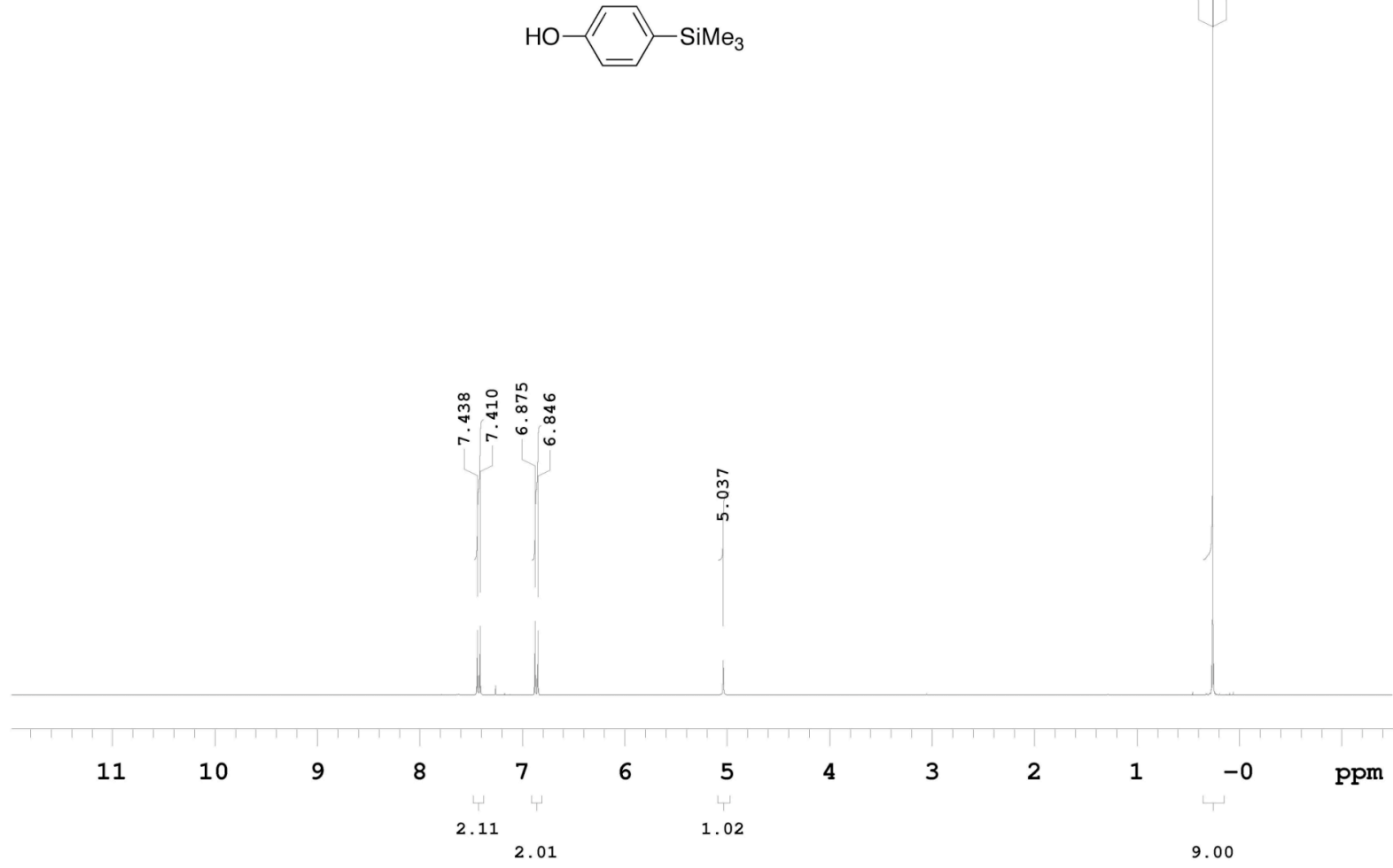




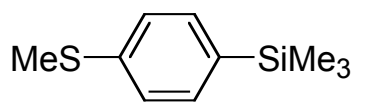

4-Trimethylsilylthioanisole (Table 2, entry 8). ${ }^{[3]}$ Following procedure A, a mixture of 4chlorothioanisole (1.0 mmol, $159 \mathrm{mg})$ KF (5 mmol, $290 \mathrm{mg})$, hexamethyldisilane (1.8 mmol, $375 \mu \mathrm{L}), \mathrm{Pd}_{2} \mathrm{dba}_{3}(0.015 \mathrm{mmol}, 13.7 \mathrm{mg}), \mathbf{1 b}(0.09 \mathrm{mmol}, 31.5 \mathrm{mg})$, and $\mathrm{H}_{2} \mathrm{O}(2 \mathrm{mmol}, 36 \mu \mathrm{L})$ in dioxane $(3 \mathrm{~mL})$ was heated to $100{ }^{\circ} \mathrm{C}$ with stirring for $24 \mathrm{~h}$. The crude product was purified via column chromatography on silica gel (1:50 ethyl acetate:hexanes) to afford the title compound as a pale yellow oil $(168 \mathrm{mg}, 86 \%) .{ }^{1} \mathrm{H}$ NMR $\left(300 \mathrm{MHz}, \mathrm{CDCl}_{3}\right): \delta 7.48(\mathrm{~d}, J=8 \mathrm{~Hz}, 2 \mathrm{H}), 7.30(\mathrm{~d}$, $J=8 \mathrm{~Hz}, 2 \mathrm{H}), 2.48(\mathrm{~s}, 3 \mathrm{H}), 0.25(\mathrm{~s}, 9 \mathrm{H}) .{ }^{13} \mathrm{C} \mathrm{NMR}\left(75 \mathrm{MHz}, \mathrm{CDCl}_{3}\right): \delta$ 139.6, 136.7, 134.0, 125.9, 15.7, -0.9. A copy of the ${ }^{1} \mathrm{H}$ NMR spectrum is provided below.

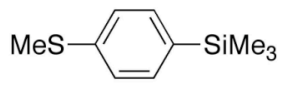




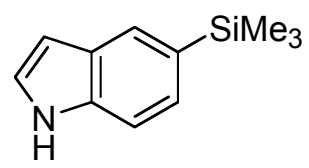

5-Trimethylsilyl-1H-indole (Table 2, entry 9). Following procedure A, a mixture of 5-chloro$1 H$-indole (1.0 mmol, $152 \mathrm{mg}), \mathrm{KF}$ (5 mmol, $290 \mathrm{mg})$, hexamethyldisilane (1.8 mmol, $375 \mu \mathrm{L})$, $\mathrm{Pd}_{2} \mathrm{dba}_{3}(0.01 \mathrm{mmol}, 9.4 \mathrm{mg}), \mathbf{1 b}(0.06 \mathrm{mmol}, 22 \mathrm{mg})$, and $\mathrm{H}_{2} \mathrm{O}$ (2 mmol, $\left.36 \mu \mathrm{L}\right)$ in dioxane (3 $\mathrm{mL}$ ) was heated to $100{ }^{\circ} \mathrm{C}$ with stirring for $24 \mathrm{~h}$. The crude product was purified via column chromatography on silica gel (1:20 ethyl acetate:hexanes) to afford the title compound as a colorless oil (159 mg, 84\%). ${ }^{1} \mathrm{H}$ NMR (300 MHz, $\left.\mathrm{CDCl}_{3}\right)$ : $\delta 8.12$ (bs, 1H), 7.85 (m, 1H), 7.42 $(\mathrm{dt}, J=9 \mathrm{~Hz}, 1 \mathrm{~Hz}, 1 \mathrm{H}), 7.35(\mathrm{~m}, 1 \mathrm{H}), 7.20(\mathrm{t}, J=3 \mathrm{~Hz}, 1 \mathrm{H}), 6.57(\mathrm{~m}, 1 \mathrm{H}), 0.32(\mathrm{~s}, 9 \mathrm{H}) .{ }^{13} \mathrm{C}$ NMR $\left(75 \mathrm{MHz}, \mathrm{CDCl}_{3}\right): \delta 136.5,130.2,127.9,126.9,126.4,124.3,110.9,102.7,-0.4$. IR (neat): 3412, 2954, 1608, 1459, 1413, 1296, 1247, 1075, 915, 837, 752, 723, 691, 621, $418 \mathrm{~cm}^{-1}$. A satisfactory elemental analysis could not be obtained for this compound. Copies of the ${ }^{1} \mathrm{H}$ and ${ }^{13} \mathrm{C}$ NMR spectra are provided below.

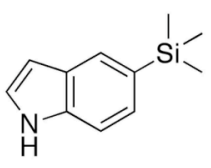




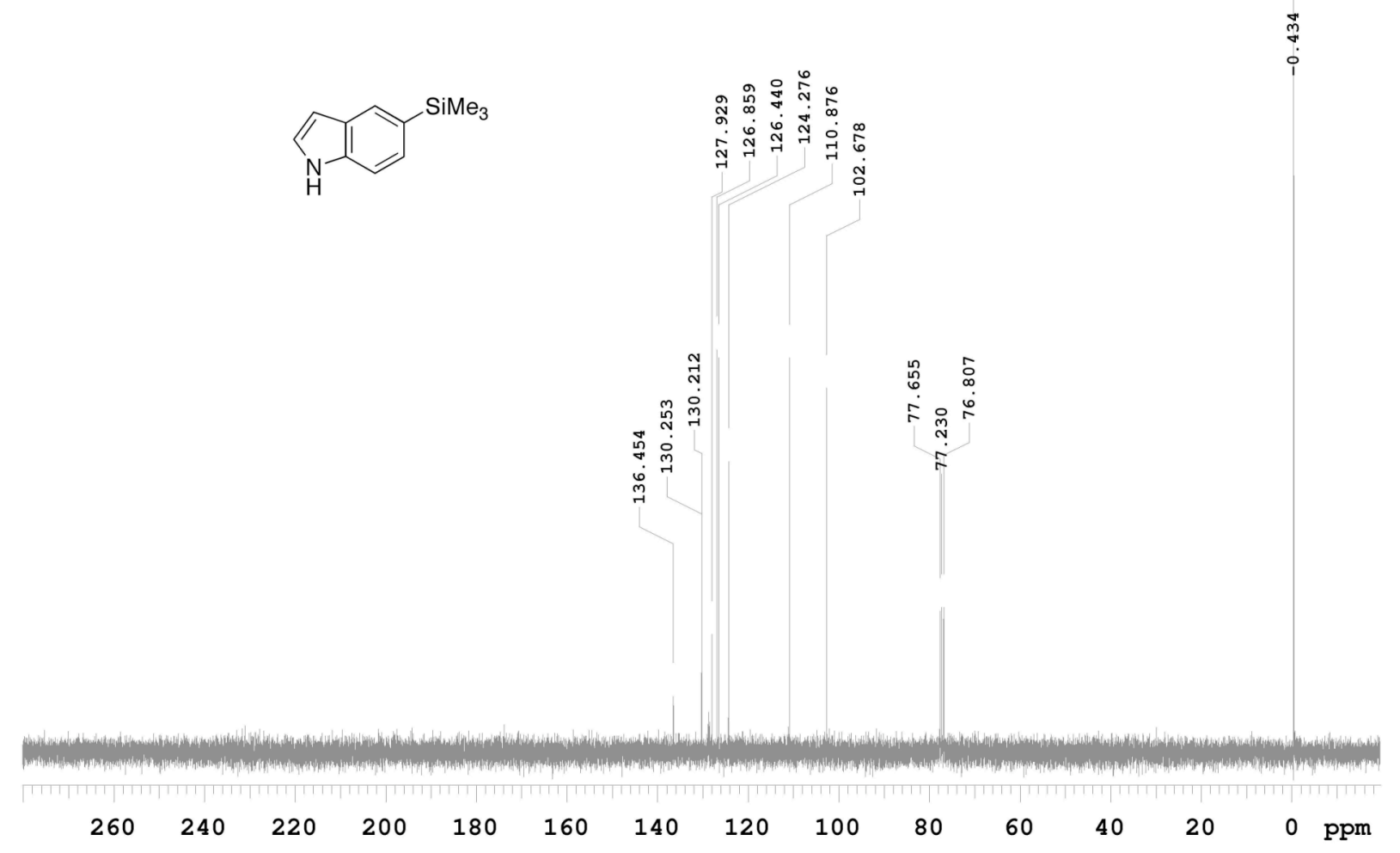<smiles>C[AsH2]c1ccc2cc3ccccc3cc2c1</smiles>

2-Trimethylsilylanthracene (Table 2, entry 10). Following procedure A, a mixture of 2chloroanthracene (1.0 mmol, $213 \mathrm{mg})$ KF (5 mmol, $290 \mathrm{mg})$, hexamethyldisilane (1.2 mmol, $250 \mu \mathrm{L}), \mathrm{Pd}_{2} \mathrm{dba}_{3}(0.015 \mathrm{mmol}, 13.7 \mathrm{mg}), \mathbf{1 b}(0.09 \mathrm{mmol}, 31.5 \mathrm{mg})$, and $\mathrm{H}_{2} \mathrm{O}(2 \mathrm{mmol}, 36 \mu \mathrm{L})$ in dioxane $(3 \mathrm{~mL})$ was heated to $100{ }^{\circ} \mathrm{C}$ with stirring for $24 \mathrm{~h}$. The crude product was purified via column chromatography on silica gel (hexanes) to afford the title compound as a pale yellow solid (173 mg, 69\%). MP: 137-139 ${ }^{\circ} \mathrm{C} .{ }^{1} \mathrm{H}$ NMR (300 MHz, $\mathrm{CDCl}_{3}$ ): $\delta 8.44$ (s, 1H), 8.40 (s, $1 \mathrm{H}), 8.18(\mathrm{~s}, 1 \mathrm{H}), 8.06-7.96(\mathrm{~m}, 3 \mathrm{H}), 7.57$ (d, $J=8.4 \mathrm{~Hz}, 1 \mathrm{H}), 7.50-7.43$ (m, 2H), 0.39 (s, 9H). ${ }^{13} \mathrm{C}$ NMR (75 MHz, $\mathrm{CDCl}_{3}$ ): $\delta$ 137.6, 134.6, 132.1, 132.0, 131.4, 129.1, 128.5, 128.4, 127.3, 126.6, 126.1, 125.6, 125.5, 125.4, -0.9. IR (neat): 2952, 1615, 1384, 1298, 1245, 1078, 961, 910, 896, 873, 834, $743 \mathrm{~cm}^{-1}$. Anal. Calcd. for $\mathrm{C}_{17} \mathrm{H}_{18} \mathrm{Si}$ : $\mathrm{C} 81.54 \%, \mathrm{H} 7.25 \%$. Found: $\mathrm{C} 81.48 \%, \mathrm{H}$ $7.10 \%$. 


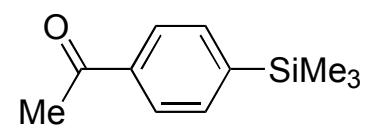

4-Trimethylsilylacetophenone (Table 4, entry 1). Following procedure B, a mixture of 4chloroacetophenone $(1.0 \mathrm{mmol}, 155 \mathrm{mg})$, LiOAc $(5 \mathrm{mmol}, 330 \mathrm{mg})$, hexamethyldisilane (1.2 mmol, $250 \mu \mathrm{L}), \mathrm{Pd}_{2} \mathrm{dba}_{3}(0.015 \mathrm{mmol}, 13.7 \mathrm{mg})$, $1 \mathbf{d}(0.09 \mathrm{mmol}, 31.5 \mathrm{mg})$, and $\mathrm{H}_{2} \mathrm{O}$ (2 mmol, 36 $\mu \mathrm{L})$ in DMF (3 mL) was heated to $100{ }^{\circ} \mathrm{C}$ with stirring for $24 \mathrm{~h}$. The crude product was purified via column chromatography on silica gel (1:50 ethyl acetate:hexanes) to afford the title compound as a colorless oil (183 mg, 95\%). ${ }^{1} \mathrm{H}$ NMR $\left(300 \mathrm{MHz}, \mathrm{CDCl}_{3}\right): \delta 7.92(\mathrm{~d}, J=8 \mathrm{~Hz}$, 2H), $7.62(\mathrm{~d}, J=8 \mathrm{~Hz}, 2 \mathrm{H}), 2.61(\mathrm{~s}, 3 \mathrm{H}), 0.29(\mathrm{~s}, 9 \mathrm{H}) .{ }^{13} \mathrm{C} \mathrm{NMR}\left(75 \mathrm{MHz}, \mathrm{CDCl}_{3}\right): \delta 198.7$, 147.5, 137.3, 133.7, 127.4, 26.9, -1.1. IR (neat): 2957, 1687, 1595, 1388, 1357, 1273, 1250, $1114,842,818,759,637 \mathrm{~cm}^{-1}$. A satisfactory elemental analysis could not be obtained for this compound. Copies of the ${ }^{1} \mathrm{H}$ and ${ }^{13} \mathrm{C}$ NMR spectra are provided below.

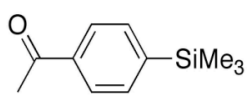

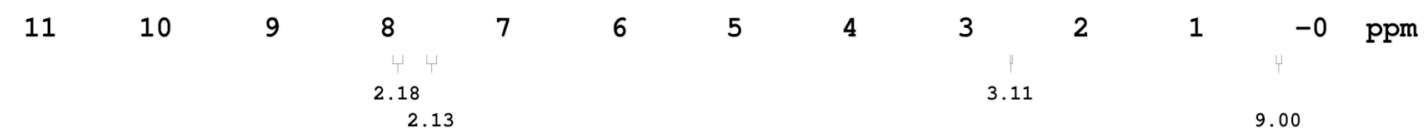




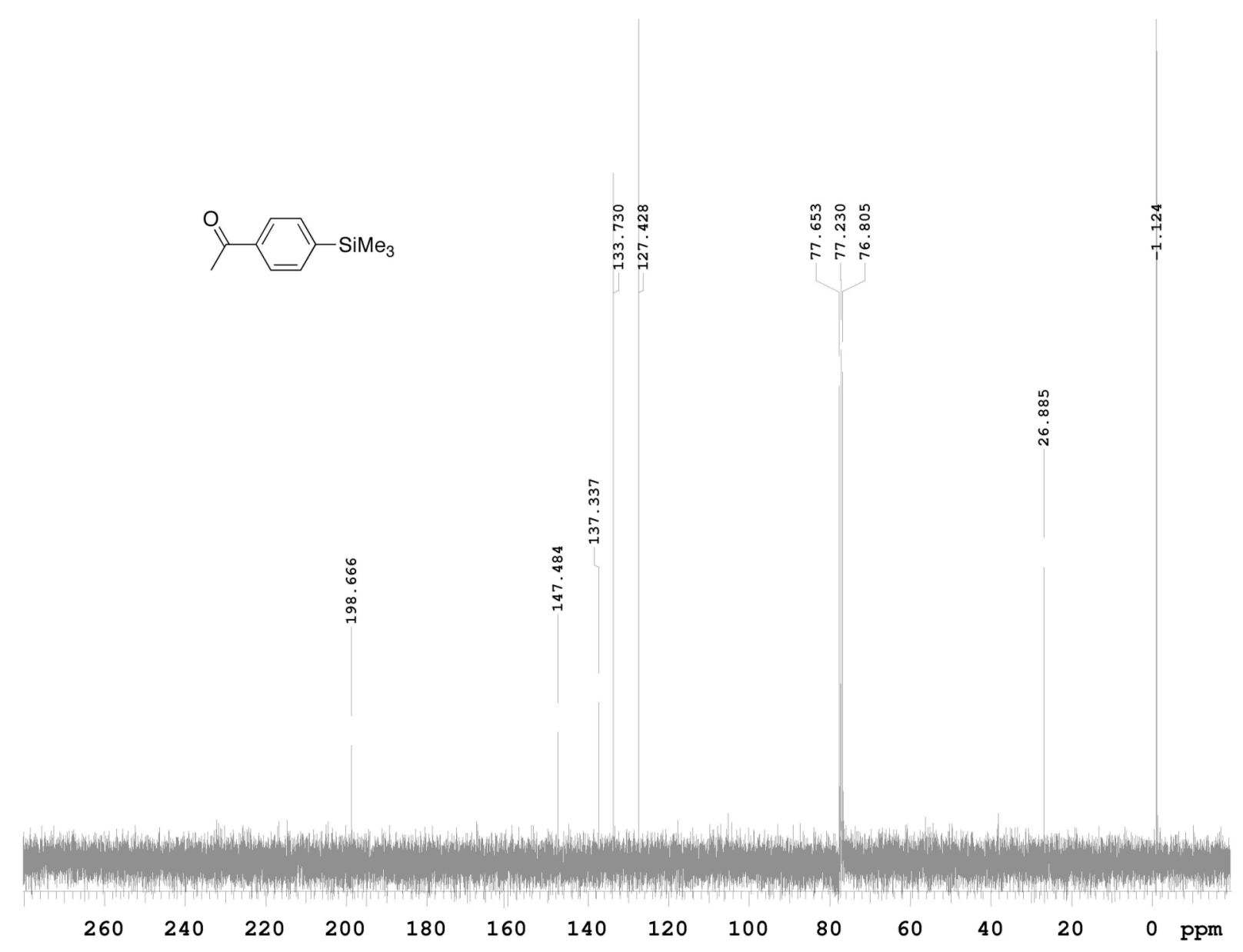<smiles>CC(C)c1ccc(C(=O)c2ccccc2)cc1</smiles>

4-Trimethylsilylbenzophenone (Table 4, entry 2). Following procedure B, a mixture 4chlorobenzophenone (1.0 mmol, $217 \mathrm{mg})$, LiOAc (5 mmol, $330 \mathrm{mg})$, hexamethyldisilane (1.2 mmol, $250 \mu \mathrm{L}), \mathrm{Pd}_{2} \mathrm{dba}_{3}(0.015 \mathrm{mmol}, 13.7 \mathrm{mg}), \mathbf{1 d}(0.09 \mathrm{mmol}, 31.5 \mathrm{mg})$, and $\mathrm{H}_{2} \mathrm{O}$ (2 mmol, 36 $\mu \mathrm{L})$ in DMF (3 mL) was heated to $100{ }^{\circ} \mathrm{C}$ with stirring for $24 \mathrm{~h}$. The crude product was purified via column chromatography on silica gel (3:100 ethyl acetate:hexanes) to afford the title compound as a colorless oil (209 mg, 82\%). ${ }^{1} \mathrm{H}$ NMR (300 MHz, $\left.\mathrm{CDCl}_{3}\right): \delta 7.83(\mathrm{~m}, 1 \mathrm{H}), 7.80$ $(\mathrm{m}, 1 \mathrm{H}), 7.78(\mathrm{~m}, 1 \mathrm{H}), 7.76(\mathrm{~m}, 1 \mathrm{H}), 7.65(\mathrm{~m}, 1 \mathrm{H}), 7.63(\mathrm{~m}, 1 \mathrm{H}), 7.58(\mathrm{~m}, 1 \mathrm{H}), 7.52-7.45(\mathrm{~m}$, 2H), $0.32(\mathrm{~s}, 9 \mathrm{H}) .{ }^{13} \mathrm{C} \mathrm{NMR}\left(75 \mathrm{MHz}, \mathrm{CDCl}_{3}\right): \delta 197.1,146.5,137.9,137.8,133.4,132.6$, 130.3, 129.2, 128.5, -1.1. IR (neat): 2955, 1660, 1597, 1448, 1388, 1317, 1280, 1249, 1103, 937, 
$922,836,728,700,662,475 \mathrm{~cm}^{-1}$. A satisfactory elemental analysis could not be obtained for this compound. Copies of the ${ }^{1} \mathrm{H}$ and ${ }^{13} \mathrm{C}$ NMR spectra are provided below.
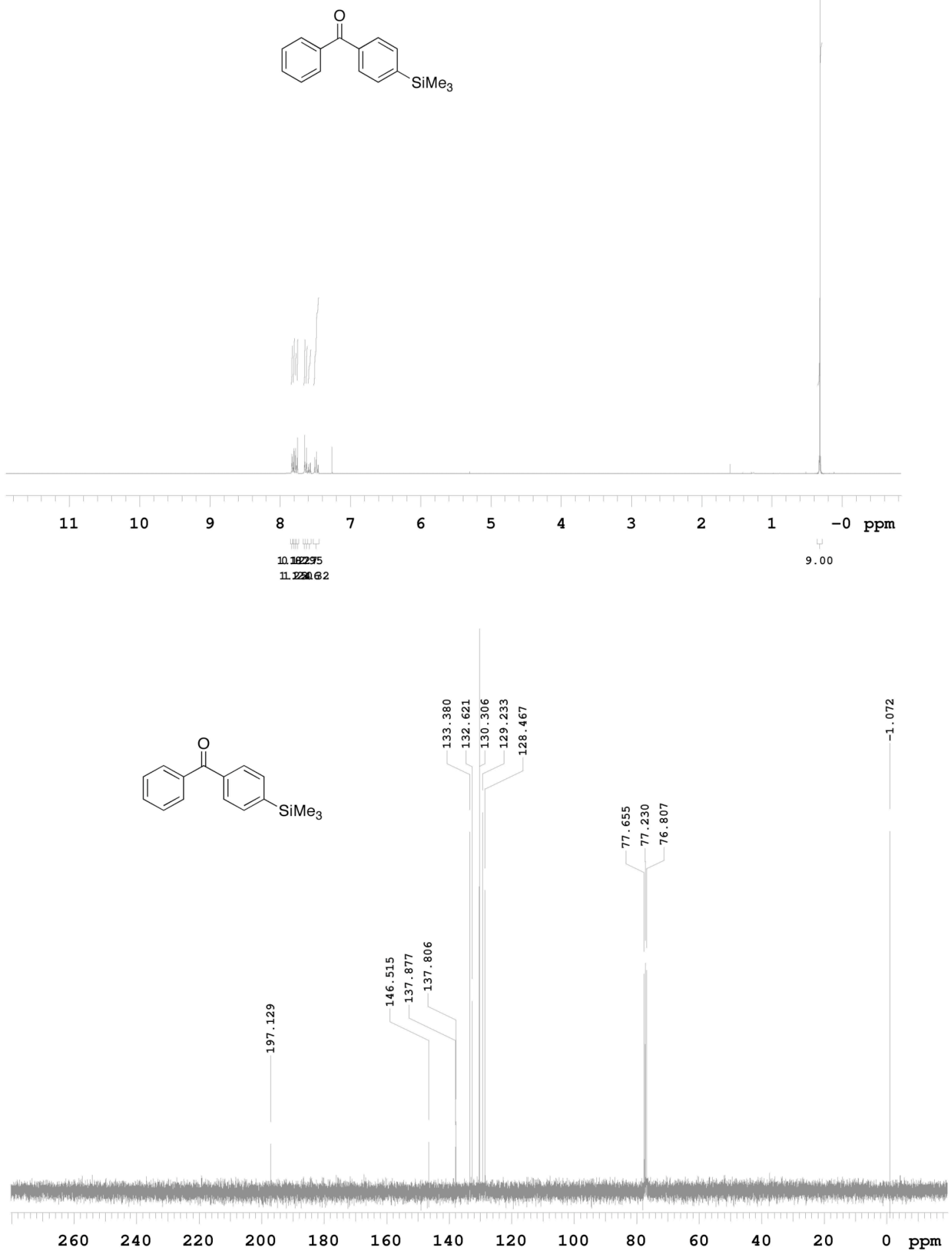
<smiles>COC(=O)c1ccc(S(C)(C)C)cc1</smiles>

Methyl 4-trimethylsilylbenzoate (Table 4, entry 3). ${ }^{[4]}$ Following procedure B, a mixture of methyl 4-chlorobenzoate (1.0 mmol, $171 \mathrm{mg})$, LiOAc (5 mmol, $330 \mathrm{mg})$, hexamethyldisilane (1.2 mmol, $250 \mu \mathrm{L}), \mathrm{Pd}_{2} \mathrm{dba}_{3}(0.015 \mathrm{mmol}, 13.7 \mathrm{mg}), 1 \mathrm{~d}(0.09 \mathrm{mmol}, 31.5 \mathrm{mg})$, and $\mathrm{H}_{2} \mathrm{O}(2$ mmol, $36 \mu \mathrm{L}$ ) in DMF (3 mL) was heated to $100{ }^{\circ} \mathrm{C}$ with stirring for $24 \mathrm{~h}$. The crude product was purified via column chromatography on silica gel (1:50 ether:hexanes) to afford the title compound as a colorless oil $(150 \mathrm{mg}, 72 \%)$. ${ }^{1} \mathrm{H}$ NMR $\left(300 \mathrm{MHz}, \mathrm{CDCl}_{3}\right)$ : $\delta 7.99$ (d, J=8 Hz, 2H), $7.59(\mathrm{~d}, J=8 \mathrm{~Hz}, 2 \mathrm{H}), 3.92(\mathrm{~s}, 3 \mathrm{H}), 0.29(\mathrm{~s}, 9 \mathrm{H}) .{ }^{13} \mathrm{C} \mathrm{NMR}\left(75 \mathrm{MHz}, \mathrm{CDCl}_{3}\right): \delta 167.6$, $147.1,133.5,130.5,128.7,52.3,-1.1$. A copy of the ${ }^{1} \mathrm{H}$ NMR spectrum is provided below.

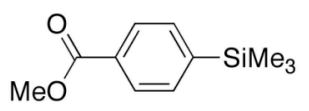

1110
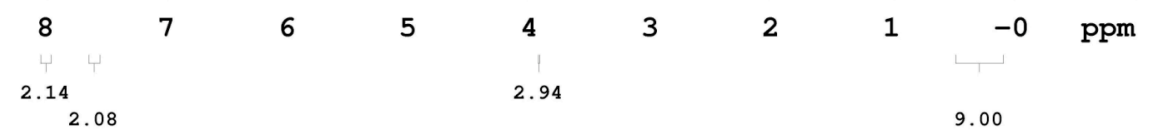


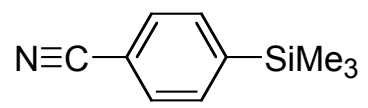

(4-Cyanophenyl)trimethylsilane (Table 4, entry 4). ${ }^{[3]}$ Following procedure B, a mixture of 4chlorobenzonitrile (1.0 mmol, $138 \mathrm{mg})$, LiOAc $(5 \mathrm{mmol}, 330 \mathrm{mg})$, hexamethyldisilane (1.2 mmol, $250 \mu \mathrm{L}), \mathrm{Pd}_{2} \mathrm{dba}_{3}(0.015 \mathrm{mmol}, 13.7 \mathrm{mg}), \mathbf{1 d}(0.09 \mathrm{mmol}, 31.5 \mathrm{mg})$, and $\mathrm{H}_{2} \mathrm{O}$ (2 mmol, 36 $\mu \mathrm{L})$ in DMF (3 mL) was heated to $100{ }^{\circ} \mathrm{C}$ with stirring for $24 \mathrm{~h}$. The crude product was purified via column chromatography on silica gel (1:50 ether:hexanes) to afford the title compound as a colorless oil (143 mg, 81\%). ${ }^{1} \mathrm{H}$ NMR (300 MHz, $\left.\mathrm{CDCl}_{3}\right): \delta 7.61(\mathrm{~s}, 4 \mathrm{H}), 0.29(\mathrm{~s}, 9 \mathrm{H}) .{ }^{13} \mathrm{C}$ NMR (75 MHz, $\mathrm{CDCl}_{3}$ ): $\delta 147.6,134.0,131.2,119.3,112.5,-1.3$. IR (neat): 2958, 2229, 1385, $1251,1103,844,821,759,714,634,552 \mathrm{~cm}^{-1}$. A copy of the ${ }^{1} \mathrm{H}$ NMR spectrum is provided below.

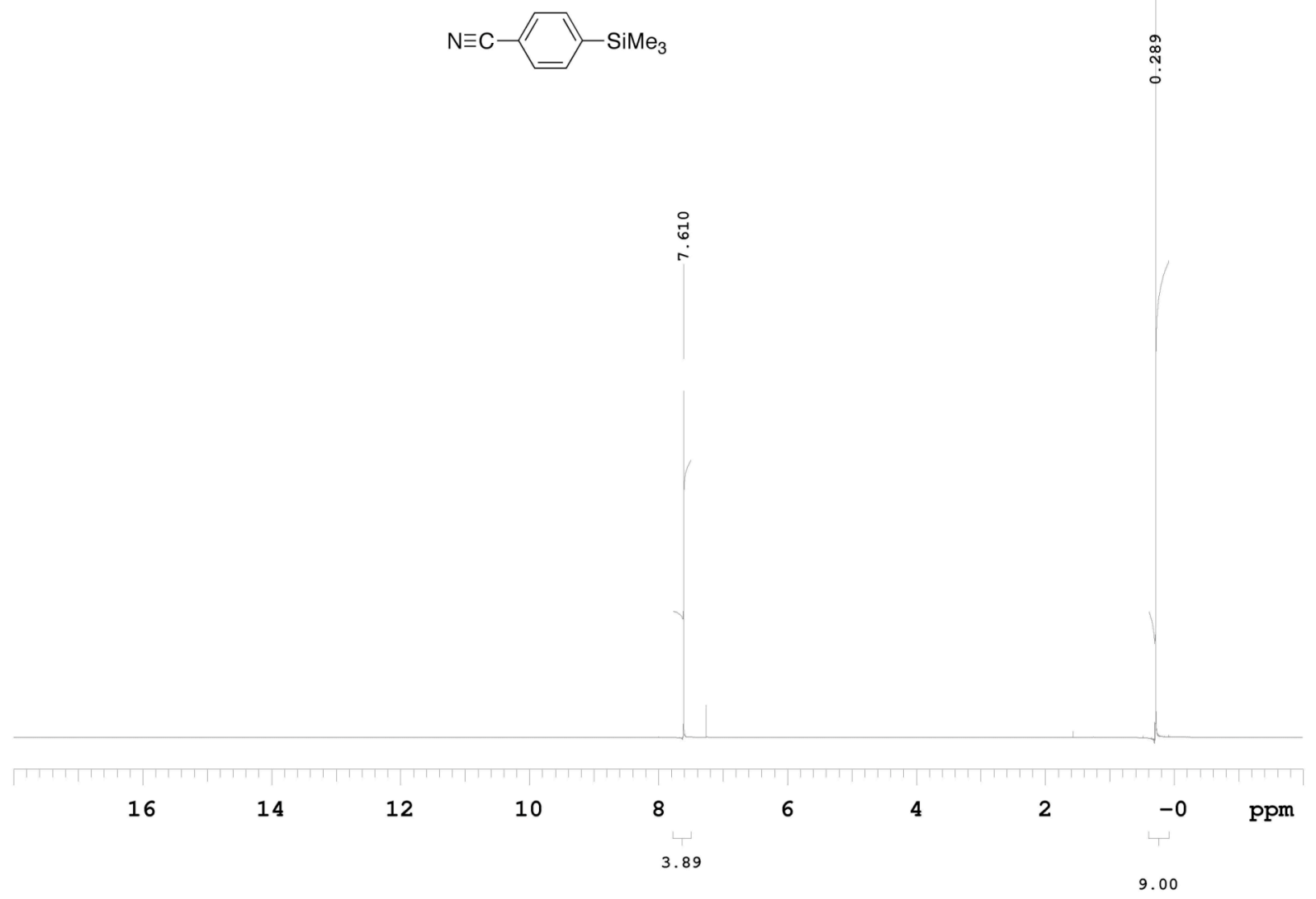


<smiles>Cc1ccc([N+](=O)[O-])cc1</smiles>

(4-Nitrophenyl)trimethylsilane (Table 4, entry 5). Following procedure B, a mixture of 1chloro-4-nitrobenzene (1.18 mmol, $186 \mathrm{mg})$, LiOAc (5 mmol, $330 \mathrm{mg}$ ), hexamethyldisilane (1.2 mmol, $250 \mu \mathrm{L}), \mathrm{Pd}_{2} \mathrm{dba}_{3}(0.015 \mathrm{mmol}, 13.7 \mathrm{mg}), \mathbf{1 d}(0.09 \mathrm{mmol}, 31.5 \mathrm{mg})$, and $\mathrm{H}_{2} \mathrm{O}$ (2 mmol, 36 $\mu \mathrm{L})$ in DMF (3 mL) was heated to $100{ }^{\circ} \mathrm{C}$ with stirring for $24 \mathrm{~h}$. The crude product was purified via column chromatography on silica gel (hexanes) to afford the title compound as a colorless solid (176 mg, 76\%). ${ }^{1} \mathrm{H}$ NMR (300 MHz, $\left.\mathrm{CDCl}_{3}\right): \delta 8.17(\mathrm{~d}, J=8 \mathrm{~Hz}, 2 \mathrm{H}), 7.68(\mathrm{~d}, J=8 \mathrm{~Hz}$, 2H), $0.32(\mathrm{~s}, 9 \mathrm{H}) .{ }^{13} \mathrm{C} \mathrm{NMR}\left(75 \mathrm{MHz}, \mathrm{CDCl}_{3}\right): \delta 150.1,134.4,122.5,-1.2$. IR (neat): 3451, $2959,1595,1515,1385,1354,1251,1112,1097,911,835,731,628,470 \mathrm{~cm}^{-1}$. A satisfactory elemental analysis could not be obtained for this compound. Copies of the ${ }^{1} \mathrm{H}$ and ${ }^{13} \mathrm{C}$ NMR spectra are provided below.

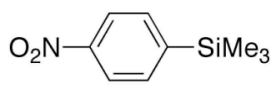

\section{6}

4

4

2

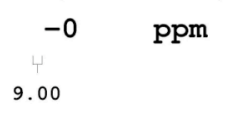




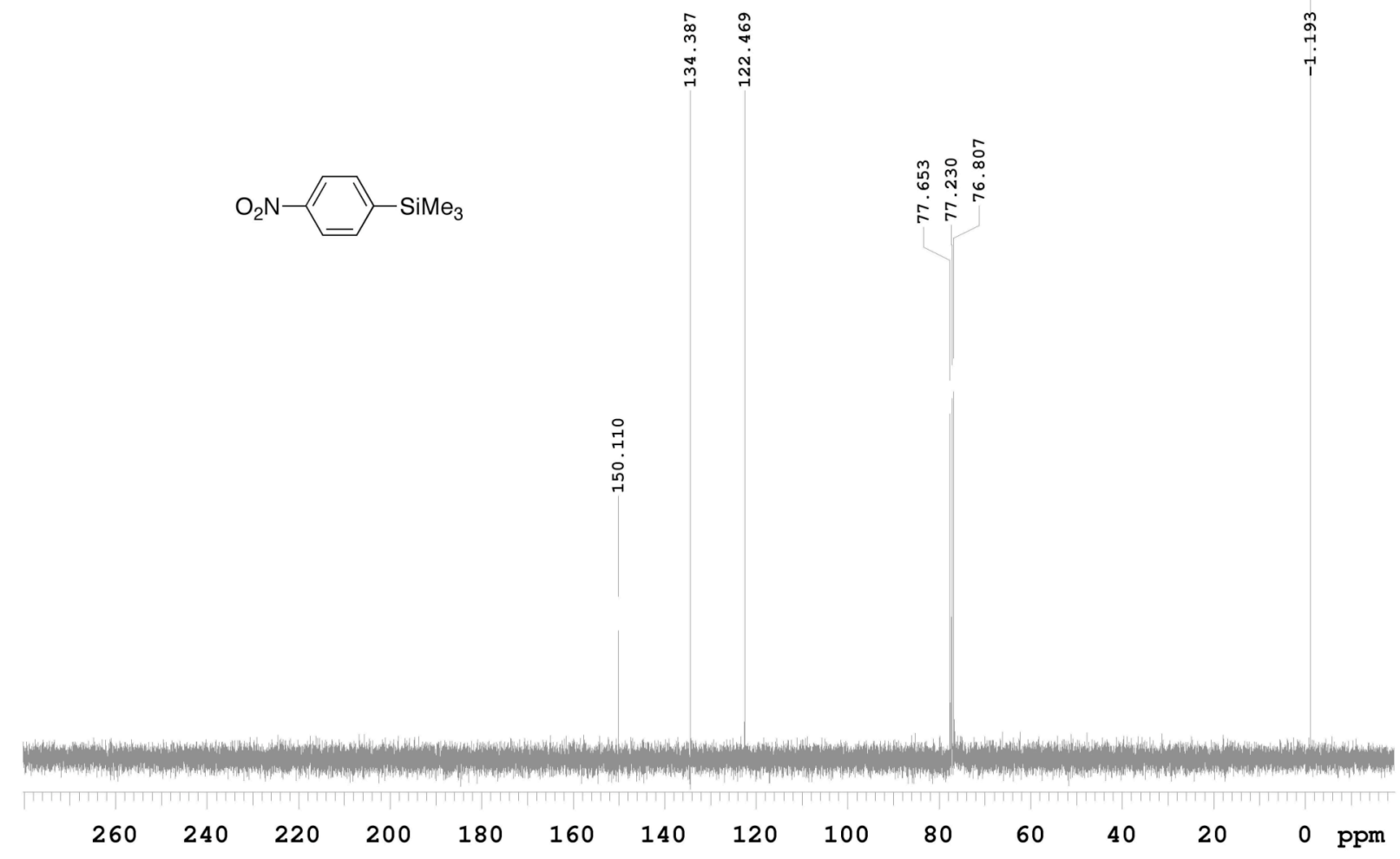

$\mathrm{SiMe}_{3}$

6-Trimethylsilylquinoline (Table 4, entry 6). Following procedure B, a mixture of 6chloroquinoline (1.0 mmol, $164 \mathrm{mg})$, LiOAc (5 mmol, $330 \mathrm{mg})$, hexamethyldisilane (1.2 mmol, $250 \mu \mathrm{L}), \mathrm{Pd}_{2} \mathrm{dba}_{3}(0.015 \mathrm{mmol}, 13.7 \mathrm{mg}), \mathbf{1 d}(0.09 \mathrm{mmol}, 31.5 \mathrm{mg})$, and $\mathrm{H}_{2} \mathrm{O}(2 \mathrm{mmol}, 36 \mu \mathrm{L})$ in DMF (3 mL) was heated to $100{ }^{\circ} \mathrm{C}$ with stirring for $24 \mathrm{~h}$. The crude product was purified via column chromatography on silica gel (1:10 ethyl acetate:hexanes) to afford the title compound as a pale yellow oil (173 mg, 86\%). ${ }^{1} \mathrm{H} \mathrm{NMR}\left(300 \mathrm{MHz}, \mathrm{CDCl}_{3}\right): \delta 8.91$ (dd, $\left.J=4 \mathrm{~Hz}, 2 \mathrm{~Hz}, 1 \mathrm{H}\right)$, $8.16(\mathrm{~d}, J=8 \mathrm{~Hz}, 1 \mathrm{H}), 8.08(\mathrm{~d}, J=8 \mathrm{~Hz}, 1 \mathrm{H}), 7.97(\mathrm{~s}, 1 \mathrm{H}), 7.85(\mathrm{dd}, J=8 \mathrm{~Hz}, 1 \mathrm{~Hz}, 1 \mathrm{H}), 7.40$ $(\mathrm{dd}, J=8 \mathrm{~Hz}, 4 \mathrm{~Hz}, 1 \mathrm{H}), 0.36(\mathrm{~s}, 9 \mathrm{H}) .{ }^{13} \mathrm{C} \mathrm{NMR}\left(75 \mathrm{MHz}, \mathrm{CDCl}_{3}\right): \delta 150.9,148.7,139.5,136.3$, 133.84, 133.75, 128.6, 127.9, 121.3, -0.9. IR (neat): 3060, 3022, 2955, 2897, 1939, 1615, 1585, 1566, 1492, 1449, 1420, 1370, 1249, 1154, 1123, 1083, 948, 876, 840, 771, 751, 694, 647, 618, 504, $478 \mathrm{~cm}^{-1}$. Anal. Calcd. for $\mathrm{C}_{12} \mathrm{H}_{15} \mathrm{NSi}$ : C 71.58\%, H 7.51\%. Found: C 71.81\%, H 7.72\%. 
$\underbrace{M e}$

2-Iodotoluene (Table 5, entry 1). ${ }^{[5]}$ Following the procedure for the one-pot sequential trimethylsilylation/iodination of electron-rich aryl chlorides, a mixture of 2-chlorotoluene (1.0 mmol, $127 \mathrm{mg}$ ), KF (5 mmol, $290 \mathrm{mg})$, hexamethyldisilane (1.2 mmol, $250 \mu \mathrm{L}), \mathrm{Pd}_{2} \mathrm{dba}_{3}(0.015$ mmol, $13.7 \mathrm{mg})$, 1 b $(0.09 \mathrm{mmol}, 31.5 \mathrm{mg})$, and $\mathrm{H}_{2} \mathrm{O}(2 \mathrm{mmol}, 36 \mu \mathrm{L})$ in 1,4-dioxane was heated to $100{ }^{\circ} \mathrm{C}$ with stirring for $24 \mathrm{~h}$. The reaction was then cooled to $\mathrm{rt}$, iodine monochloride (1.5 mmol, 1.0 M solution in $\mathrm{CH}_{2} \mathrm{Cl}_{2}$ ) was added via syringe, and the reaction was stirred for $4 \mathrm{~h}$ at rt. After workup, the crude product was purified by column chromatography on silica gel (hexanes) to afford the title compound as a colorless oil (111 mg, 51\%). ${ }^{1} \mathrm{H} \mathrm{NMR} \mathrm{(300} \mathrm{MHz,}$ $\left.\mathrm{CDCl}_{3}\right): \delta 7.81(\mathrm{~d}, J=8 \mathrm{~Hz}, 1 \mathrm{H}), 7.24(\mathrm{dd}, J=5 \mathrm{~Hz}, 1 \mathrm{~Hz}, 2 \mathrm{H}), 6.90-6.80(\mathrm{~m}, 1 \mathrm{H}), 2.43(\mathrm{~s}, 3 \mathrm{H})$. ${ }^{13} \mathrm{C}$ NMR $\left(75 \mathrm{MHz}, \mathrm{CDCl}_{3}\right): \delta 141.6,139.1,130.0,128.4,127.6,101.4,28.4$. Anal. Calcd. for $\mathrm{C}_{7} \mathrm{H}_{7} \mathrm{I}: \mathrm{C} 38.56 \%, \mathrm{H} 3.24 \%$. Found: C $38.56 \%, \mathrm{H} 3.29 \%$.<smiles>COc1ccc(I)cc1</smiles>

4-iodoanisole (Table 5, entry 2). ${ }^{[6]}$ Following the procedure for the one-pot sequential trimethylsilylation/iodination of electron-rich aryl chlorides, a mixture of 4-chloroanisole (1.0 mmol, $143 \mathrm{mg}$ ), KF (5 mmol, $290 \mathrm{mg})$, hexamethyldisilane (1.2 mmol, $250 \mu \mathrm{L}), \mathrm{Pd}_{2} \mathrm{dba}_{3}(0.015$ mmol, $13.7 \mathrm{mg}), \quad \mathbf{1 b}(0.09 \mathrm{mmol}, 31.5 \mathrm{mg})$, and $\mathrm{H}_{2} \mathrm{O}(2 \mathrm{mmol}, 36 \mu \mathrm{L})$ in 1,4-dioxane was heated to $100{ }^{\circ} \mathrm{C}$ with stirring for $24 \mathrm{~h}$. The reaction was then cooled to rt, iodine monochloride (1.5 mmol, 1.0 M solution in $\mathrm{CH}_{2} \mathrm{Cl}_{2}$ ) was added via syringe, and the reaction was stirred for $4 \mathrm{~h}$ at rt. After workup, the crude product was purified by column chromatography on silica gel (hexanes) to afford the title compound as a white solid (188 mg, 80\%). MP: 50-52 ${ }^{\circ} \mathrm{C}$ (lit. 50-53 $\left.{ }^{\circ} \mathrm{C}\right) .{ }^{1} \mathrm{H} \mathrm{NMR}\left(300 \mathrm{MHz}, \mathrm{CDCl}_{3}\right): \delta 7.56(\mathrm{~d}, J=9 \mathrm{~Hz}, 2 \mathrm{H}), 6.69(\mathrm{~d}, J=9 \mathrm{~Hz}, 2 \mathrm{H}), 3.78(\mathrm{~s}, 3 \mathrm{H})$. ${ }^{13} \mathrm{C}$ NMR $\left(75 \mathrm{MHz}, \mathrm{CDCl}_{3}\right): \delta 159.6,138.4,116.5,82.9,55.5$. Anal. Calcd. for $\mathrm{C}_{7} \mathrm{H}_{7} \mathrm{IO}: \mathrm{C}$ $35.92 \%$, H $3.01 \%$. Found: C $36.12 \%$, H $3.02 \%$. 


\section{References}

(1) Itami, K.; Terakawa, K.; Yoshida, J.; Kajimoto, O. J. Am. Chem. Soc. 2003, 125, 6058-6059.

(2) Hevesi, L.; Dehon, M.; Crutzen, R.; Lazarescu-Grigore, A. J. Org. Chem. 1997, 62, 20112017.

(3) van Walree, C. A.; Lauteslager, X. Y.; van Wageningen, A. M. A.; Zwikker, J. W.; Jenneskens, L. W. J. Organomet. Chem. 1995, 496, 117-125.

(4) Tobisu, M.; Kita, Y.; Chatani, N. J. Am. Chem. Soc. 2006, 128, 8152-8153.

(5) Bailey, W. F.; Cioffi, E. A.; Wiberg, K. B. J. Org. Chem. 1981, 46, 4219-4225.

(6) Wan, S.; Wang, S. R.; Lu, W. J. Org. Chem. 2006, 71, 4349-4352. 